\title{
Research on Task Planning Problem of Satellite-Ground Time Synchronization
}

\author{
Zhongshan Zhang ${ }^{1, a}$, Weijie Sun ${ }^{2, b}$, and Jungang Yan ${ }^{1, c}$ \\ ${ }^{1}$ National University of Defense Technology, Changsha 410073, China \\ ${ }^{2}$ Beijing Satellite Navigation Center, Beijing 100094, China \\ a zhongshanzhang@outlook.com, bzszhang@nudt.edu.cn, c1499490163@qq.com
}

\begin{abstract}
Keywords: satellite-ground time synchronization; ground station scheduling; multi-objective problem; evolutionary algorithm; global navigation satellite system.
\end{abstract}

\begin{abstract}
The task planning of satellite-ground time synchronization (SGTSTP) is a complex multi-objective ground station scheduling problem. In this paper, we establish the problem formulation and analyze the optimization objectives. Based on the complexity analysis, we design a solving framework based on decomposition, which divides the plan horizon into many un-interactional plan periods and execute task planning in each period. In algorithm selection, the MOGA and NSGA2 are selected. At last, two different scale scenarios are designed and the computational result shows that more periods can get better result but spend more time, besides, the MOGA has better performance in efficiency and effect than NSGA2, however NSGA2 can get more solutions in solving SGTSTP.
\end{abstract}

\section{Introduction}

Satellite-ground time synchronization(SGTS) operation, which is executed by building the satellite-ground link, is core operation in global navigation satellite system (GNSS).[1] The task planning of satellite-ground time synchronization (SGTSTP) is to schedule ground antennas to build the communication link with visible satellites for executing the satellite-ground time synchronization task (for brevity, "task"). SGTSTP is a ground station scheduling problem, which is a very complex problem due to its over-constrained nature. The small-scale ground station scheduling problem can be solved manually, but as the scale increasing, the manually solving or brute force search is unrealistic and a computational intelligent method must be used.

At present, the scheduling problem about ground station mainly includes satellite ranging scheduling problem (SRSP) [2], multi-satellite tracking telemetry and command (tt\&c) scheduling problem(MuSTSP) [3] and satellite-ground data transmission scheduling problem (SGDRSP) [4] and so on, all above problems are combination optimization problem. In comparison with other ground station problems, SGTSTP not only has common features of scheduling problem, but also has own characteristic because of special problem background. Firstly, this is a multi-objective optimization problem. Secondly, the decision variables number is more than other scheduling problem.

Most of ground station scheduling problem are single objective optimization problem in current research. But the ground station scheduling problem always don't have only one objective in practice, some researchers also carried out a lot of researches on the multi-objective ground station scheduling problem. In the references [5-7], xhafa defined four main objectives as follows that would compose the fitness function: access window fitness, communication clashes fitness, communication time requirement fitness and ground station usage fitness. Besides, the four fitness value calculating formulas are given. But, though xhafa took in consideration four objectives, the solving method is still to combine four objectives with one objective and use single objective optimization algorithm.

In this paper, we use multi-objective evolutionary algorithm to solve SGTSTP. The remainder of this paper is organized as follows. In the second section we build a general mathematical model. In the third section, we establish atomic task mixed integer programming model based decomposition, the framework of model and coding form are also designed. In the fourth section, we selected MOGA 
and NSGA2 to solve this problem. At last, two different scale computational experiences are designed to test the model and algorithm.

\section{Problem Definition and Analysis}

Description of SGTS. To provide higher position accuracy for users, GNSS must remain higher time standard and space standard. Maintaining time standard mainly rely on atomic clock on satellite, which has inevitable error due to complex space environment and atomic clock autologous error. In general, the clock error is main influencing factor of UERE (user equivalent range error) [8]. Therefore, to improve position accuracy, it is necessary to use measure data between antennas and satellites to predict accurate clock error, which users can receive to get more accurate position. The satellite-ground time synchronization operation is a processing of collecting measure data.

The SGTS operation flow is showed in Fig. 1. There is a benchmark time T0 in ground station and a time $T 1$ in satellite, through by communicating between antenna and satellite the ground station gets the difference of $T 0$ and $T 1$, which called clock error. The main control station (MCS) collects all the clock errors, and makes a clock error prediction, which is broadcasted to users for positioning.

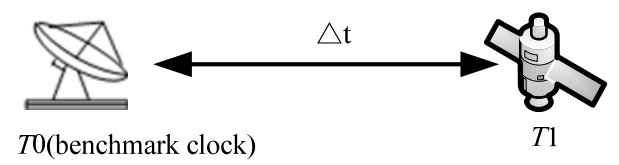

Fig. 1 Operation flow of SGTS

Problem Formulation. The related parameter and set are defined as follows.

$t_{\text {begin }}$ : plan begin time

$t_{\text {end }}$ : plan end time

planHorizon: planHorizon $=\left[t_{\text {begin }}, t_{\text {end }}\right]$

$S$ : set of satellites, $S=\{1, \ldots, s, \ldots\}$, the set element number is $n_{s}$.

$M$ : set of antennas, $M=\{1, \ldots, m, \ldots\}$, the set element number is $n_{m}$.

$V_{(s, m)}:$ set of time windows,

$$
V_{(s, m)}=\bigcup_{h=1, \cdots, H_{s m}}\left[t w_{s m}^{\text {start }(h)}, t w_{s m}^{e n d}(h)\right], s \in S, m \in M
$$

The system is designed so that each satellite $s \in S$ is visible from at least one station $m \in M$ within the planHorizon, that is, $s \in S, m \in M$ such that $V_{(s, m)} \neq \varnothing$.

$t$ : SGTS task, which refers to the operation that require ground-to-satellite communications, it can be defined by tetrad as follows.

$\{$ antenna, satellite, begin time, end time $\}=\left\{t_{s}, t_{m}, t^{\text {start }}, t^{\text {end }}\right\}$

$T$ : set of tasks, it also can be defined as follow.

$$
T=\bigcup_{i=1, \ldots, I_{I m}}\left[t_{s m}^{\operatorname{sart}(i)}, t_{s m}^{\operatorname{end}(i)}\right]=\bigcup_{l=1, \ldots, L_{m}}\left[t_{m}^{\operatorname{sart}(l)}, t_{m}^{\operatorname{end}(l)}\right]=\bigcup_{o=1, \ldots, O_{s}}\left[t_{s}^{\operatorname{sart}(o)}, t_{s}^{\operatorname{end}(o)}\right], s \in S, m \in M
$$

besides, $t_{m}^{\text {end }(l)} \leq t_{m}^{\text {start }(l+1)}, t_{s}^{\text {end }(o)} \leq t_{s}^{\text {start }(o+1)}$.

$\sigma_{m}$ : set-up time of antenna, $m \in M$

$\varphi_{m}$ : service ability of antenna, $m \in M$

$v_{s}:$ service ability of satellite, $s \in S$

$\eta_{t}$ : the shortest task duration

The problem constrains are defined as follows. 
Set-up time constrain: Whenever a ground station $m$ processes two consecutive services with two distinct satellites $i$ and $j$, the ground station requires a setup time $\sigma_{m}$ in order to point its antenna. During such a setup time, no further transmissions can occur. The mathematical statement is as follow. In this paper, we assume the set-up time is zero.

$$
l \leq L_{m}, t_{m}^{\text {start }(l+1)}-t_{m}^{\text {end }(l)} \leq \sigma_{m}, \forall m \in M .
$$

Task duration constrain: The execute time length for every task $t$ must greater than $\eta_{t}$. The mathematical statement is as follow.

$$
s \in S, t_{s m}^{\text {end }(i)}-t_{s m}^{\text {start }(i)} \geq \eta_{t}, \forall i=1, \cdots, I_{s m}, m \in M .
$$

Time window constrain: ground station could communicate with a satellite only when the satellite lies within the transmitting horizon of the ground station (called time window or visibility window). In general, this happens periodically within a planning horizon. That means there are several communication chances between a ground station and a satellite. The mathematical statement is as follow.

$$
\forall i=1, \cdots, I_{s m}, m \in M, s \in S, \quad \exists 1 \leq h \leq H_{s m}, \quad t_{s m}^{\text {end }(i)} \leq t w_{s m}^{\text {end }(h)}, t_{s m}^{\text {start }(i)} \geq t w_{s m}^{\text {start }(h)}
$$

Service ability of antenna constrain: The number of antenna can provide communication service at any time. This value is always 1 . The setting value is 1 in this paper, that is, $\varphi_{m}=1, \forall m \in M$

Service ability of satellite constrain: The number of satellite can build communication service at any time. This value is always 1 . The setting value is 1 in this paper, that is, $v_{s}=1, \forall s \in S$

Optimization Objectives Definition. Firstly, we use symbol $m D s$ as the average task duration of satellite $s$ and use $m I_{s}$ as average interval of neighborhood task of satellite $s$. The calculation formula of $m D s$ and $m I_{s}$ is as follow.

$$
\begin{aligned}
m D_{s}= & \frac{\sum_{o=1}^{O_{s}}\left(t_{s}^{\text {end }(o)}-t_{s}^{\text {start }(o)}\right)}{O_{s}} \\
m I_{s} & =\frac{\sum_{o=1}^{O_{s}}\left(t_{s}^{\text {start }(o+1)}-t_{s}^{\text {end }(o)}\right)}{O_{s}}
\end{aligned}
$$

In GNSS, all satellites want to maximize $m D_{s}$ and minimize $m I_{s}$, the aim of task planning is to satisfy all satellites' requirement. However, as the system controller, they must take the system balance into consideration except satisfying requirement of each satellite. So we define the $p_{\text {duration }}$ and $p_{\text {interval }}$ as the system objectives. The calculation formulas of two objectives' are as follows.

$$
\min \quad p_{\text {duration }}=\frac{\sqrt{\frac{\sum_{s \in S}\left(m D_{s}-\sum_{s \in S} m D_{s} / n_{s}\right)^{2}}{n_{s}}}}{\sum_{s \in S} m D_{s} / n_{s}} .
$$

$$
\min p_{\text {interval }}=\sqrt{\frac{\sum_{s \in S}\left(m I_{s}-\sum_{s \in S} m I_{s} / n_{s}\right)^{2}}{n_{s}} \times \frac{\sum_{s \in S} m I_{s}}{n_{s}}} .
$$




\section{Model}

Solve Framework. SGTSTP is a complex oversubscribed scheduling problems (OSP) which has several difficulties as follows.

a) The control variables scale is huge. Not only resources and executing time of each task are included, task number and task duration also must be assigned, which is difficult to control.

b) A whole consideration in planning is necessary because there are some influences and effect among tasks. The evaluation of planning result is not an easy summation of each task's revenue, but is a whole evaluation of all tasks'.

c) This is a typical multi-objective optimization problem, and these objectives are mutually exclusive. Therefore, the previous algorithm about ground station scheduling problem is inapplicable and new algorithms must be proposed.

Taking above complexities and difficulties into consideration, we proposed a preprocessing model based on decomposition. As show in Fig. 2, the model divides the whole plan horizon into many equal plan periods, meanwhile, all time window data are also divided into every plan period (or, for brevity, "period"). We regard the indivisible time window in each period as a pre-planning task (called "atomic task"), then we execute atomic task planning in each period, therefore, the task planning problem changed into a combinatorial optimization problem of atomic tasks, which distinctly is a $0-1$ programming problem. In addition, each divided time window can't be divided again.

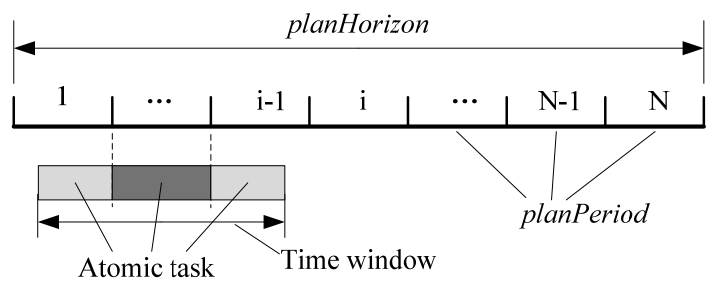

Fig. 2 Sketch of preprocessing model based on decomposition

After determining the atomic tasks in each period, a splicing operation should be executed to combine tasks in neighborhood periods because two neighborhood tasks may be combined into one task. We use symbol $\sum \oplus$ to indicate splicing operation, the principle that two tasks can be spliced is as follow, $t 1$ and $t 2$ are respectively used to express the two neighborhood atomic tasks.

$$
\left(t 1_{s}=t 2_{s}\right) \cap\left(t 1_{m}=t 2_{m}\right) \cap\left(t 1^{\text {end }}=t 2^{\text {start }}\right)
$$

On the basis of preprocessing model based on decomposition, a task planning framework based on decomposition is proposed, as show in Fig. 3. The operation of dealing after planning is to remove those tasks that violate constrains to insure tasks enforceability. Need not point out that all genetic operations, such as selection, crossover and mutation and so on, are executed independently in each period and there is no influence in different periods.

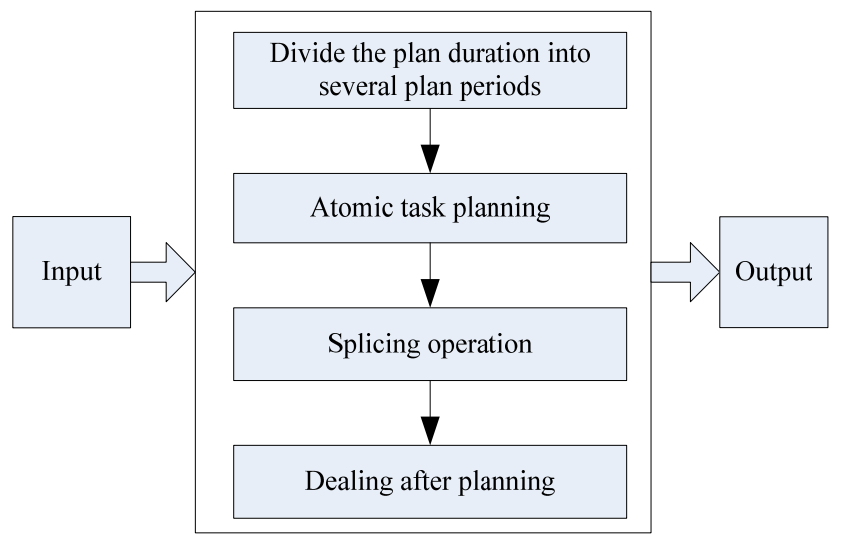

Fig. 3 Solving framework based on decomposition 
Modeling. We assume that the plan horizon can be divided into $N$ periods, and use $n t_{s m}^{i}$ express the atomic task of period $i$.

We define $x_{s m}^{i}$ mark whether the $n t_{s m}^{i}$ is selected, then we know

$$
x_{s m}^{i}=\left\{\begin{array}{l}
1, n t_{s m}^{i} \text { is selected } \\
0, \text { others }
\end{array}\right.
$$

Then the final planning scheme $T$ can be calculated as follow formula.

$$
T=\sum_{i=1}^{p} \oplus x_{s m}^{i} \times n t_{s m}^{i}
$$

From the above, we designed a atomic task planning model based decomposition, which is a $0-1$ programming model, the objectives and constrains are as follows.

Objectives:

$$
\min p_{\text {duration }}
$$

$$
\min p_{\text {interval }} \times \lambda
$$

Subject to:

$$
\begin{aligned}
& \sum_{m \in M} x_{s m}^{i} \leq 1 \quad \forall 1 \leq i \leq N, s \in S \\
& \sum_{s \in S} x_{s m}^{i} \leq 1 \quad \forall 1 \leq i \leq N, m \in M
\end{aligned}
$$

Explain about objective: The objectives are still the two objectives in last section, but for a more intuitionistic effect show in diagram, we use parameter $\lambda$ to make an exchange on objective $p_{\text {interval }}$. Need to notice that the value $10^{-7}$ is a fit value after several computational tests.

Coding Operation. We choose a 0-1 coding form. We firstly arrange all atomic tasks in each period according a fixed sort, the subcode digit number is equal to number of atomic task. When the $i$-th atomic task is selected $\left(x_{s m}{ }^{i}=1\right)$, the subcode digit is 1 , or else 0 . Then, the part-code in each period is a $0-1$ code, all part-code combines into one individual code. Coding form is showed in Fig. 4.

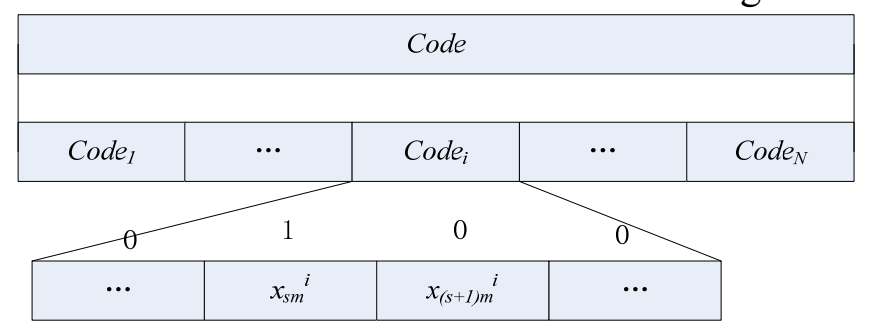

Fig. 4 Sketch of code formation

\section{Algorithms for SGTSTP}

SGTSTP is a typical multi-objective problem and doesn't have optimal solution like in single objective optimization problem, its optimal solution is the Pareto optimal solutions (called Pareto front). In recent years, multi-objective evolutionary algorithm (MOEA) is continually used to solve 
practical problem. In this paper, we select MOEA to solve SGTSTP. In MOEAs, the MOGA (multi-objective genetic algorithm) and NSGA2 (non-dominated sorting genetic algorithm II) are two famous algorithms and are both successfully applied in some practical application.

MOGA. MOGA[9] is proposed in 1993, and has a huge practical application market due to its usability. The algorithm flow is as follow.

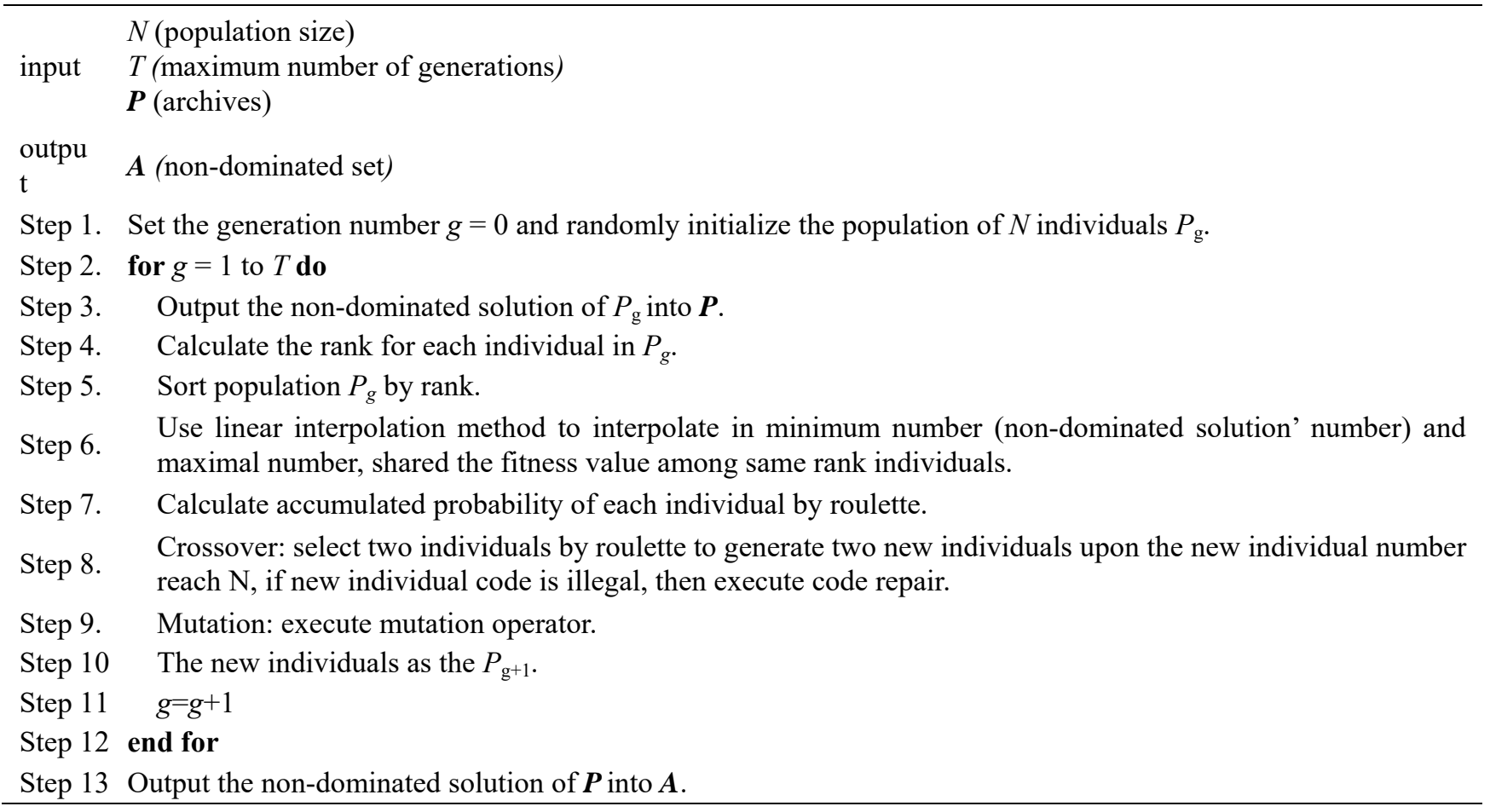

NSGA2. NSGA2[10] adopts another algorithm architecture different from MOGA. NSGA2 is a popular and frequently-used algorithm, and often deemed as preferred algorithm in solving practical problem and comparison object with other MOEAs.

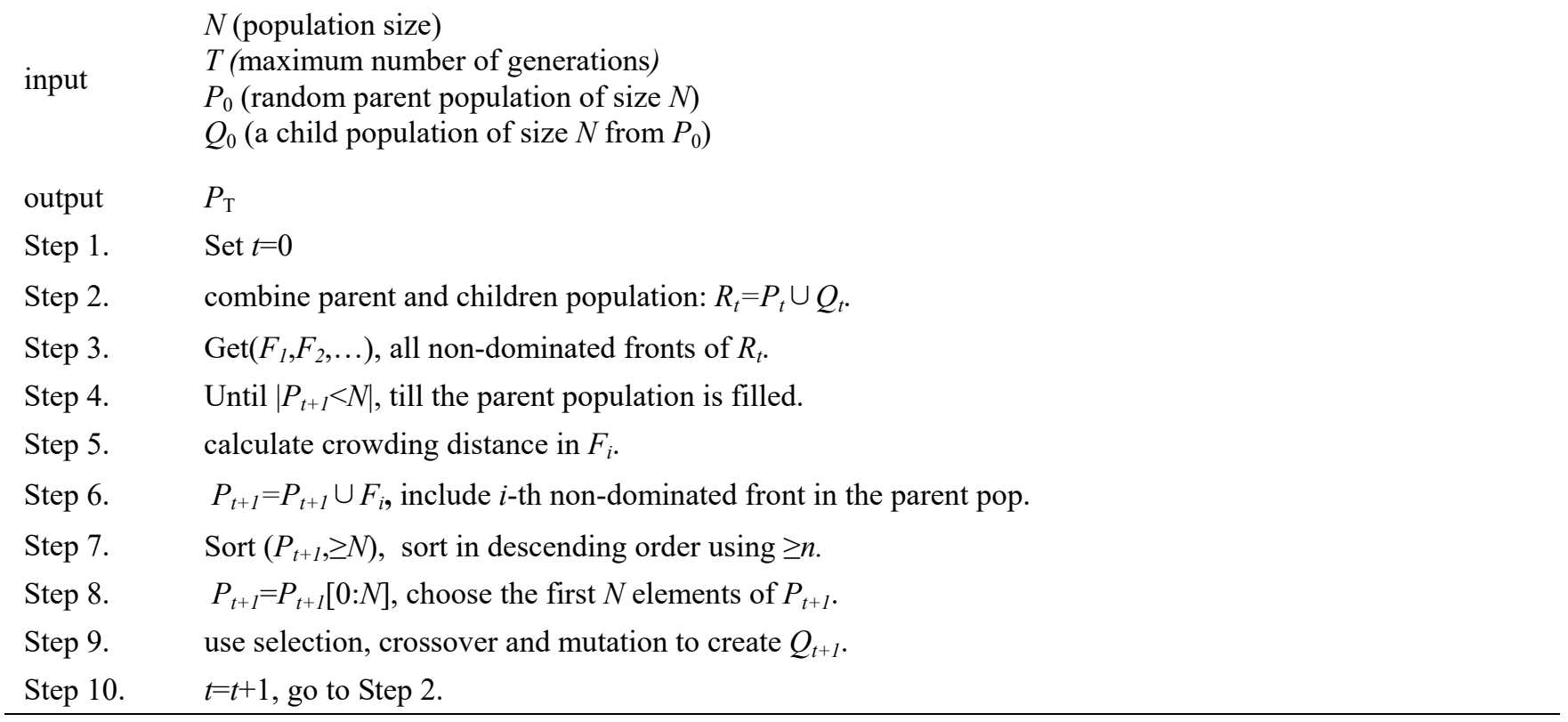

\section{Computational Experience}

Test Bed Description. A computational experience was designed based on Compass navigation system of China, and the simulation parameters are designed as 
Table 1. 
Table 1 Simulation parameters

\begin{tabular}{|l|l|l|}
\hline Parameters & Values & remark \\
\hline$t_{\text {begin }}$ & $2016-01-0100: 00: 00$ UTC & - \\
\hline$t_{\text {end }}$ & $2016-01-0800: 00: 00$ UTC & - \\
\hline$\eta_{t}$ & $30 \mathrm{~min}$ & - \\
\hline planPeriod & $30 \mathrm{~min}, 45 \mathrm{~min}, 60 \mathrm{~min}$ & \\
\hline Crossover probability & 0.7 & For MOGA and NSGA2 \\
\hline Mutation probability & 0.3 & For MOGA and NSGA2 \\
\hline Population number & 100 & For MOGA and NSGA2 \\
\hline Iterations steps & 500 & For MOGA and NSGA2 \\
\hline
\end{tabular}

To verify effectiveness of model and algorithms, we design two different scale instances, in which has different number antennas but same number satellites, as Table 2 and Table 3.

Table 2 Simulation instances

\begin{tabular}{|c|c|c|c|}
\hline Instances & Antennas Number & Antennas & Navigation constellation \\
\hline Small scale & 1 & BJC-1 & 24 MEO(Medium Earth Orbit) satellites, which \\
Medium scale & 3 & BJC-1 & belongs Walker24/3/2 constellation with 55 \\
& 3 & KHC-1 & inclination and $21500 \mathrm{~km}$ orbital altitude \\
& & SNC-1 & \\
\hline
\end{tabular}

Table 3 Parameters of antennas

\begin{tabular}{|c|c|c|c|c|}
\hline Station & Antenna & Elevation angle & Azimuth angle & Location (longitude, latitude) \\
\hline BJC & BJC-1 & {$[15,90]$} & {$[0,360]$} & $(116.388,39.929)$ \\
\hline KHC & KHC-1 & {$[15,90]$} & {$[0,360]$} & $(75.98,39.455)$ \\
\hline SNC & SNC-1 & {$[15,90]$} & {$[0,360]$} & $(109.505,18.243)$ \\
\hline
\end{tabular}

Computational Results Analysis. We executed algorithms for 10 times and make a result statistic as follow diagrams.

From Fig. 5, we can find that MOGA has better performance than NSGA2 in solving small scale instance. The same conclusion can be given from Fig. 7 in medium scale instance. But NSGA2 can get more solutions than MOGA, which will be more useful in practice.

From Fig. 6, we can find that the result has better performance when the plan period is smaller in small scale instance. But from Fig. 10 we can find that the algorithm time is increasing as the plan period decreasing. The same conclusion can be given from Fig. 8 in medium scale instance.

From Fig. 9, we can find that there is an obviously better result when the antennas number increasing, but as show in Fig. 10, the time also increasing sharply as the antennas number increasing.
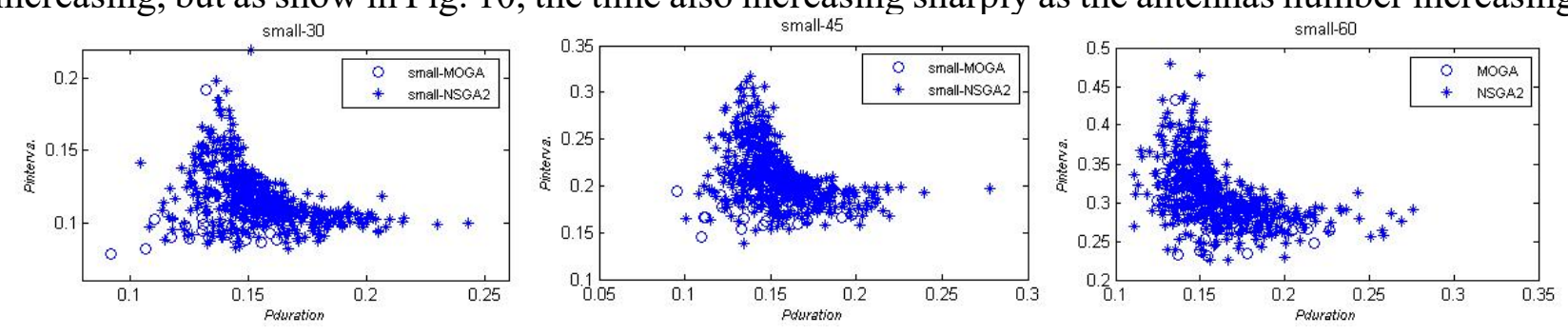

Fig. 5 Algorithm performance under different planPeriod in small scale
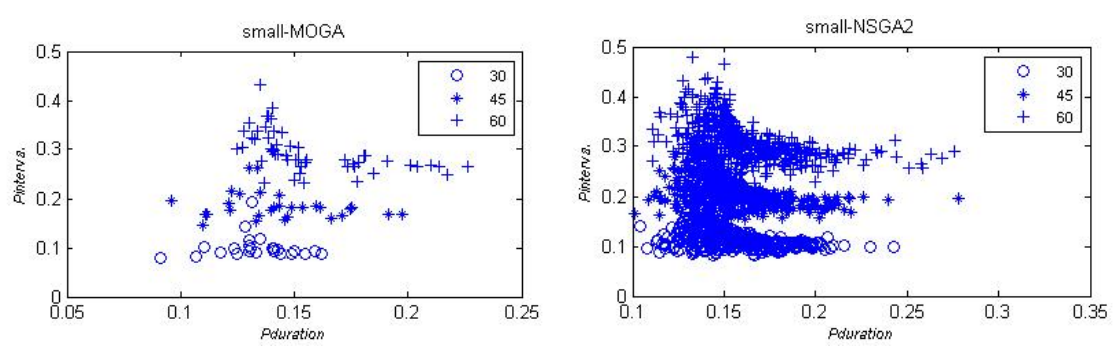

Fig. 6 Performance of different algorithms in small scale 

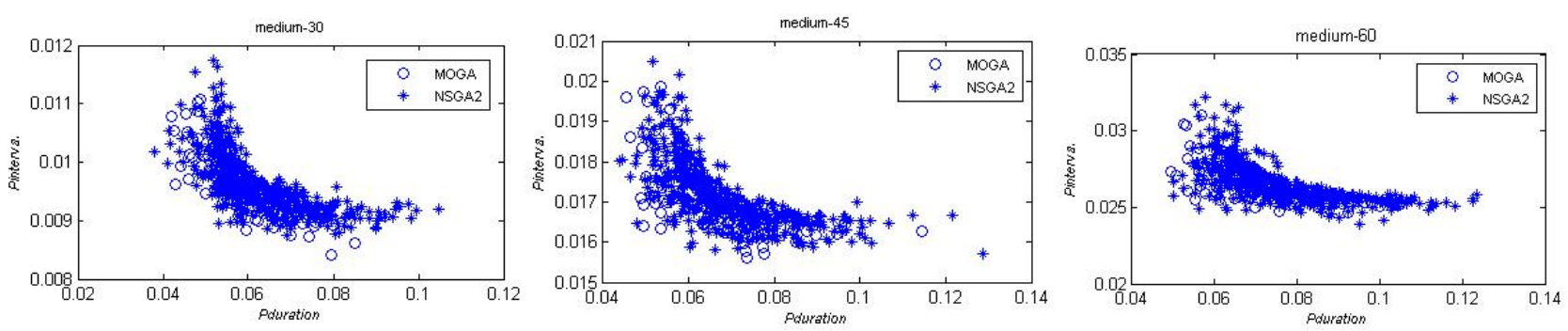

Fig. 7 Algorithm performance under different planPeriod in medium scale
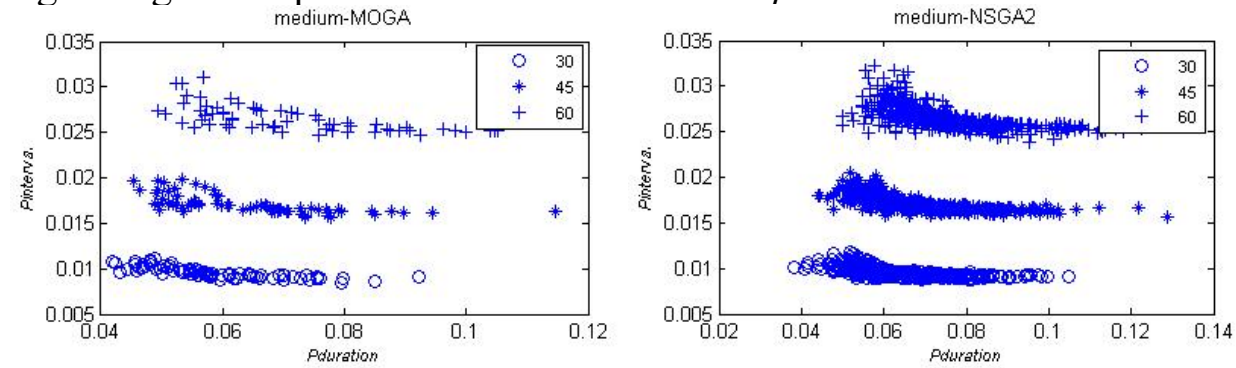

Fig. 8 Performance of different algorithms in medium scale
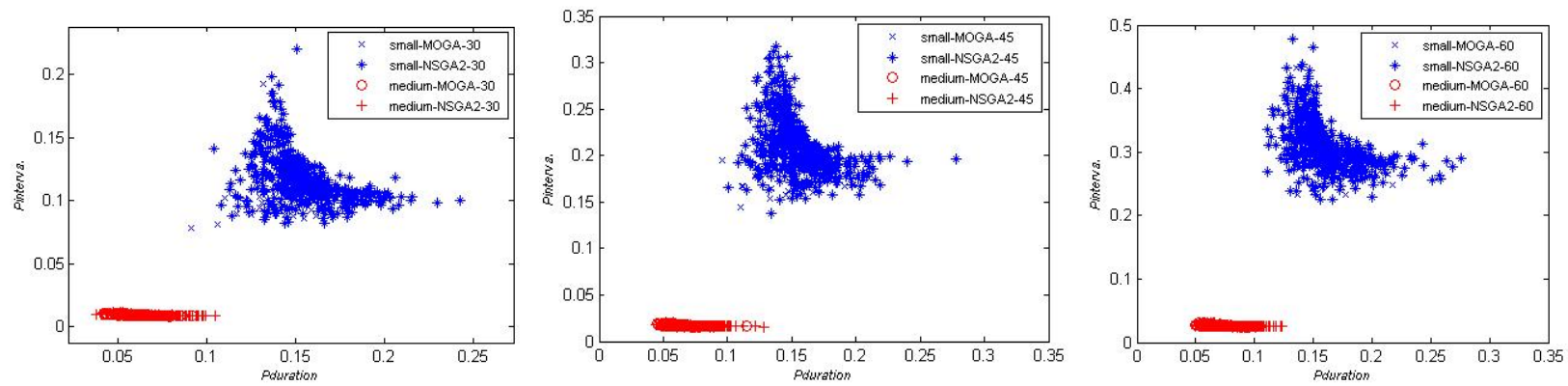

Fig. 9 Performance of different algorithm in each instance

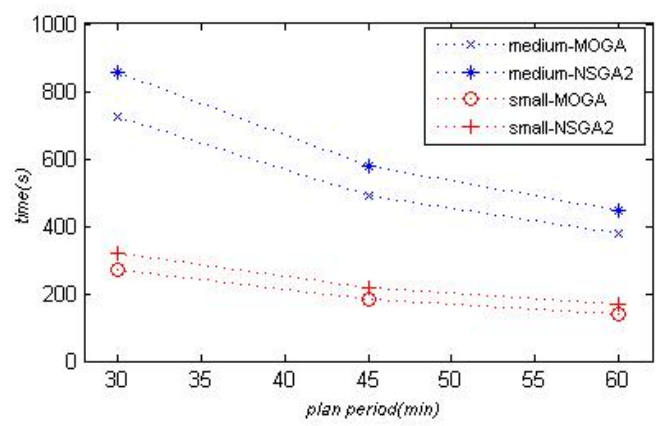

Fig. 10 Algorithm time

\section{Conclusion}

In this paper, we carried out the research on SGTSTP, which is a complex multi-objective ground station scheduling problem. Firstly, we build the problem formulation and analyze the optimization objectives. Then we design a solving framework based on decomposition, in which we changed the task planning problem into a 0-1 programming problem. And then the MOGA and NSGA2 are selected to solve this multi-objective optimization problem. At last, two different scale simulation scenarios are designed based on the Compass navigation system of China, and the computational result shows that more periods can get better result but spend more time, besides, the MOGA has better performance in efficiency and effect than NSGA2 in solving SGTSTP. In the future, we will continue researching on the multi-objective ground station scheduling problem, and the new and effective multi-objective algorithms and scheduling problem with more complex constrains will be focused emphasis. 


\section{Acknowledgment}

This work was supported by the National Natural Science Foundation of China (71201171,61473301,71501179).

\section{References}

[1] T. Shusen. The engineering of satellite navigation and positioning(second edition). Beijing: National Defense Industry Press (2010).

[2] L. Barbulescu, J.-P. Watson, L. D. Whitley, and A. E. Scheduling space-ground communications for the air force satellite control network [J]. Journal of Scheduling, Vol. 7 (2004), p. 7-34.

[3] X. D. Ling, W. K. Zhu, J. M. Wu, and X. Y. Wu. Research of multi-satellite tt\&c scheduling problem [J]. Applied Mechanics and Materials, Vol. 263 (2013), p. 476-484.

[4] L. Yunfeng. Research on Satellite-Ground Station Data Transmission Scheduling Models and Algorithms. Doctor, National University of Defense Technology, National University of Defense Technology, Changsha (2008).

[5] F. Xhafa, X. Herrero, A. Barolli, and M. Takizawa. A Tabu Search Algorithm for Ground Station Scheduling Problem. 2014 IEEE 28th International Conference on Advanced Information Networking and Applications, Victoria, BC, Canada (2014).

[6] F. Xhafa, X. Herrero, A. Barolli, L. Barolli, and M. Takizawa. Evaluation of struggle strategy in Genetic Algorithms for ground stations scheduling problem [J]. Journal of Computer and System Sciences, Vol. 79 (2013), p. 1086-1100.

[7] A. Lala, V. Kolici, F. Xhafa, X. Herrero, and A. Barolli. On Local vs Population-based Heuristics for Ground Station Scheduling. 2015 Ninth International Conference on Complex, Intelligent, and Software Intensive Systems, Blumenau, Brazil (2015).

[8] E. D. Kaplan and C. J. Hegarty. Understanding GPS: Principles and Applications(second edition). America: Artech House (2007).

[9] F. C. M and F. P. J. Genetic algorithms for multi-objective optimization: Formulation, discussion and generalization. Proceeding of the Fifth Internationl Conference on Genetic Algorithms, San Mateo and California (1993), p. 416-423.

[10]D. K, P. A, A. S, and e. al. A fast and elitist multi-objective genetic algorithms: NSGA2 [J]. IEEE Transactions on Evolutionary Computation, Vol. 6 (2002), p. 182-197. 